\title{
Intraoperative Severe Anaphylaxis Due to Gelofusine in a Patient Undergoing Elective Prostatectomy: Case Report
}

\author{
Amad Hania
}

\begin{abstract}
Anaphylaxis due to colloid plasma expanders is a recognized but rare life-threatening complication in patients. It is seen most commonly in patients in perioperative setting, with no history of allergy, whom receive colloid plasma expanders for the first time to maintain adequate blood pressure during surgery. A case of a 64-year-old male undergoing elective prostatectomy, who develops severe anaphylaxis and urticaria, 30 minutes following gelofusine infusion, is presented in this report. The diagnosis of anaphylaxis secondary to gelofusine was confirmed by skin prick testing in the immunology department.
\end{abstract}

Keywords: Anaphylaxis; Colloid; Gelofusine; Skin prick testing; Urticaria; Allergy

\section{Introduction}

Colloid plasma expanders are widely used in surgery and in resuscitation of hypovolemic patients [1]. They provide intravascular volume expansion and help in the immediate management of patients in severe shock. The colloid plasma expander gelofusine (succinylated gelatin) is a recognized cause of perioperative anaphylaxis. Anaphylaxis to Gelofusine and other colloid plasma expanders carries an estimated incidence of $0.07-0.15 \%$ [2]. This allergic reaction is usually a type- 1 hypersensitivity reaction which is IgE-mediated and causes the production of antibodies through prior sensitisation, although in most cases they occur without any previous exposure. Currently the diagnosis of gelofusine allergy is made by skin prick and/or intradermal testing, a procedure that itself carries a risk of allergic reaction. Colloid solutions are used commonly in fluid resuscitation in hypovolemic patients; however, they can

Manuscript accepted for publication August 26, 2014

General Medicine Scheme, St James Hospital, Dublin, Ireland. Email: haniaa@tcd.ie

doi: http://dx.doi.org/10.14740/jmc1924w rarely result in life-threatening reaction which requires prompt recognition and treatment.

\section{Case Report}

A 64-year-old Caucasian male patient, undergoing elective radical prostatectomy, developed anaphylactic shock during the operation. The patient was previously well, with no history of allergies or drug reactions. The patient was undergoing surgery for early-stage prostate cancer. The patient had previous surgery 50 years ago for perforated nasal antrum under general anesthetic. There were no documented issues during previous anesthetic. The only medication that the patient was taking was tamsulosin for prostatism. The patient underwent induction with general anesthetic at 14:30 with propofol, fentanyl, and rocuronium (a neuromuscular blocker). At 14:50 an epidural analgesia bupivocaine was commenced. At 15:00 gelofusine infusion was commenced, and $30 \mathrm{~min}$ later, the patient developed profound hypotension, with systolic blood pressure dropping from 120 to $50 \mathrm{~mm} \mathrm{Hg}$. The hypotension was unresponsive to fluid resuscitation and also to phenylephrine and ephedrine boluses. The patient did not develop any increase in his peak airway pressure, suggestive of no respiratory compromise and remained well ventilated at the operating table. The patient required noradrenaline infusion during surgery to maintain adequate mean arterial blood pressure. The patient during theater was treated for likely anaphylaxis and received IV chlorphenamine $10 \mathrm{mg}$ and hydrocortisone $200 \mathrm{mg}$ (Fig. 1 ). The patient remained hemodynamically stable with mean arterial pressure of $60 \mathrm{~mm} \mathrm{Hg}$. During surgery, the patient was found to have global erythematous papular rash mainly in trunk and limbs consistent with urticaria; however, there was no evidence of angioedema such as lip or tongue swelling and there was no mucosal involvement. The patient underwent successful radical prostatectomy; however, he still required inotropic support and remained in post-anesthesia care unit (PACU) overnight and was slowly weaned of all inotropes. Serum mast cell tryptase $1 \mathrm{~h}$ after the event demonstrated a level of 85 (ng/ $\mathrm{mL})$ which dropped to $32(\mathrm{ng} / \mathrm{mL}) 6 \mathrm{~h}$ later. The raised levels of mast cell tryptase were consistent with mast cell degranulation, which is strongly suggestive of an allergic reaction. The patient's urticaria resolved and was discharged by the surgical team following full recovery from radical prostatectomy. The 


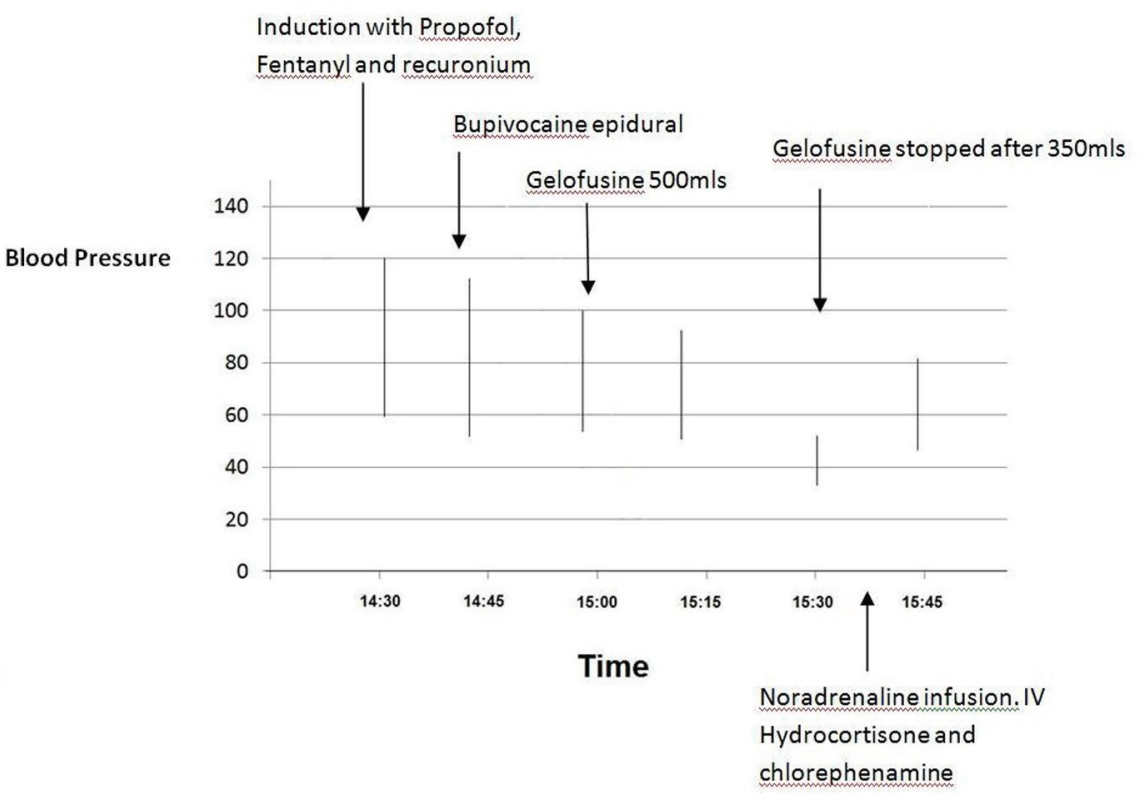

Figure 1. Time chart showing the changes in blood pressure over time, in response to different agents used perioperatively.

patient was subsequently referred to immunology department and underwent allergy skin testing to fentanyl, propofol, rocuronium, chlorhexidine, betadine and bupivocaine which were all negative. Specific IgE to chlorhexidine and latex was also negative. The patient however developed a positive skin prick testing response to gelofusine with a wheal diameter of $4 \mathrm{~mm}$ and also a positive test to intradermal skin testing to gelofusine with a flare reaction of $>20 \mathrm{~mm}$ at $15 \mathrm{~min}$ mark (Table 1). The patient was informed of the results and was told to avoid gelofusine and other colloid plasma expanders permanently and also advised to obtain a MedicAlert bracelet.

\section{Discussion}

Plasma expanders play an important role in perioperative period and also during volume resuscitation in critically ill patients outside the perioperative setting. Anaphylactic reaction to colloid volume expanders is a rare but recognized risk. There is controversy regarding the merits of using colloid versus crystalloids in the setting of fluid resuscitation. A systematic review of 37 randomized control trials comparing the use of colloids versus crystalloids for fluid resuscitation, in critically ill patients, showed that resuscitation with colloids was associated with an increased absolute risk of mortality of $4 \%$. The review did not support the continued use of colloids for volume replacement in critically ill patients [3].

Adverse reactions to colloid volume expanders tend to occur within 10 min of commencing the infusion and require early and frequent monitoring [1]. Allergy to gelatine has also been associated with anaphylactic reactions to Measles-MumpsRubella (MMR) vaccination, with one-quarter of children suffering from anaphylaxis to the MMR vaccine demonstrating

Table 1. The Results of Allergy Tests of Possible Causative Agents Used in Theater

\begin{tabular}{ll}
\hline Skin test & Result \\
\hline Gelofusine & Positive intradermal test (flare $>20 \mathrm{~mm}$ ) \\
& Positive skin prick (wheal diameter $4 \mathrm{~mm}$ ) \\
Fentanyl & Negative \\
Propofol & Negative \\
Rocuronium & Negative \\
Chlorhexidine & Negative \\
Betadine & Negative \\
Bupivocaine & Negative \\
Latex & Negative \\
\hline
\end{tabular}


IgE antibody production to gelatine [4]. Other than gelofusine, there have also been other documented cases of anaphylactic reactions to other gelatine containing volume expanders such as Haemaccel [5]. It had also been shown that cross-reactivity between Haemaccel and gelofusine existed [5]. As such, there must be a high level of suspicion, and care taken with the use of other gelatine based colloids, once a diagnosis of anaphylaxis is made, to any one of these agents.

The diagnosis of anaphylaxis to colloids can be very difficult in a perioperative setting, and also in the setting of fluid resuscitation in a critically ill patient. This severe reaction usually results in cardiorespiratory compromise and subsequently shock. In a critically ill patient this can be as a result of a wide range of possible etiology including the patients, own underlying disorder that is requiring fluid resuscitation, such as infection or trauma. In a perioperative setting, such as in this case reported here, there is a wide variety of possible agents that may have resulted in anaphylaxis. In this case, the two most likely culprit agents were, gelofusine and rocuronium, which have been shown to cause anaphylaxis in a perioperative setting [6]. However the patient required testing to further possible agents used in theater, such as latex, propofol, fentanyl, bupivociane, etc. As in the setting of resuscitating a critically ill patient, there was a wide range of other possible etiology causing shock perioperatively; however, the evidence of urticaria and elevated serum tryptase was indicative of mast cell degranulation and strongly suggested anaphylaxis as the likely diagnosis. Serum tryptase levels usually peak $1 \mathrm{~h}$ after an allergic reaction, and can remain elevated for several days. However, this test is not specific in recognizing the causative agent. Gelofusine allergy is usually diagnosed by skin prick testing and intradermal testing, a procedure that itself carries a risk of allergic reaction. Less invasive methods of confirming gelofusine hypersensitivity has been described by using in vitro basophil activation test, in which blood tests are analyzed with flow CD63 surface expression. In the series of subjects, the basophil activation test for gelofusine allergy had a sensitivity of $100 \%$ and a specificity of $87.5 \%$ [7]. This offers a safe and reliable method in diagnosing gelofusine sensitivity in patients with high clinical suspicion of gelofusine-induced anaphylaxis.

\section{References}

1. Mishler JMt. Synthetic plasma volume expanders--their pharmacology, safety and clinical efficacy. Clin Haematol. $1984 ; 13(1): 75-92$.

2. Ring J, Messmer K. Incidence and severity of anaphylactoid reactions to colloid volume substitutes. Lancet. 1977;1(8009):466-469.

3. Schierhout $\mathrm{G}$, Roberts I. Fluid resuscitation with colloid or crystalloid solutions in critically ill patients: a systematic review of randomised trials. BMJ. 1998;316(7136):961964.

4. Pool V, Braun MM, Kelso JM, Mootrey G, Chen RT, Yunginger JW, Jacobson RM, et al. Prevalence of antigelatin $\operatorname{IgE}$ antibodies in people with anaphylaxis after measles-mumps rubella vaccine in the United States. Pediatrics. 2002;110(6):e71.

5. Russell WJ, Fenwick DG. Anaphylaxis to Haemaccel and cross reactivity to Gelofusin. Anaesth Intensive Care. 2002;30(4):481-483.

6. Bhananker SM, O'Donnell JT, Salemi JR, Bishop MJ. The risk of anaphylactic reactions to rocuronium in the United States is comparable to that of vecuronium: an analysis of food and drug administration reporting of adverse events. Anesth Analg. 2005;101(3):819-822, table of contents.

7. Apostolou E, Deckert K, Puy R, Sandrini A, de Leon MP, Douglass JA, Rolland JM, et al. Anaphylaxis to Gelofusine confirmed by in vitro basophil activation test: a case series. Anaesthesia. 2006;61(3):264-268. 